\title{
Epidemiology of Depression in Rheumatoid Arthritis, Systemic Lupus Erythematosus, and Systemic Sclerosis
}

\author{
Elham Rajaei ${ }^{1}$, Zahra Shalbaf ${ }^{1}$, Masoumeh Nazarinasab ${ }^{2}$ and Zeinab Deris Zayeri ${ }^{1,{ }^{*}}$ \\ ${ }^{1}$ Clinical Research Development Unit, Golestan Hospital, Ahvaz Jundishapur University of Medical Sciences, Ahvaz, Iran \\ ${ }^{2}$ Department of Psychiatry, Golestan Hospital, Ahvaz Jundishapur University of Medical Sciences, Ahvaz, Iran \\ "Corresponding author: Golestan Hospital Clinical Research Development Unit, Ahvaz Jundishapur University of Medical Sciences, Ahvaz, Iran. Email: \\ zeynabderisgenetice@gmail.com
}

Received 2019 February 20; Revised 2019 May 16; Accepted 2019 May 23.

\begin{abstract}
Background: Depression is a major disabling factor around the world. Most of the mental disorders immediately affect the inner mood and cognitive aspect of individuals. Therefore, hiding the depression can delay the early diagnosis and, consequently, a proper treatment in the patients.

Objectives: We designed the study to determine the association between rheumatoid diseases and depression.

Methods: This is a descriptive - analytic study which was performed on 354 patients referred to Rheumatology Clinic of Ahvaz, a province in south west of Iran in 2016. Then demographic information, depression, quality of life and social support were completed based on standardized questionnaires filled by the patients.

Results: The prevalence of depression in rheumatoid arthritis (RA), systemic lupus erythematous (SLE) and systemic sclerosis (SSc) patients was $61.63 \%, 73.52 \%$ and $60 \%$, respectively. There is a meaningful relationship between depression, age and duration of disease in SSc patients. There is a significant relationship between depression and gender in SLE and RA patients. There is a significant relationship between depression and the degree of education of SLE patients. There is a meaningful relationship between depression and quality of life, social support and corticosteroid consumption.

Conclusions: The prevalence of depression is very high in all three diseases, which is equal in terms of quality of life, social support and corticosteroid usage in all three diseases. Psychiatric disorders such as depression are common in rheumatic patients.
\end{abstract}

Keywords: Depression, Social Support, Psychiatry, Rheumatoid Arthritis, Systemic Lupus Erythematosus, Scleroderma

\section{Background}

Mood is an emotional state that affects the perception of the world in the individuals. Mood disorders include a wide range of disorders, which constitute the pathological mood and related disturbances of the dominant clinical features. These disorders were identified as "affective disorders" in some previous versions of the diagnostic and statistical manual of psychiatric disorders (DSM), but later the term "mood disorders" was preferred. Mood disorders can best be described by syndrome (rather than as distinct diseases). Because these disorders are grouped from signs and symptoms that might last weeks to months; the symptoms have a marked difference in thenormal function of the individual; they are usually recurrent, and often a periodic or cyclic pattern exists. The mood may be normal, elevated, or depressed (1). Rheumatoid arthritis (RA) is a common and chronic disease that affects $0.8 \%$ of the population (2). Recent evidence showed that the preva- lence of depression in patients with SLE is approximately $47 \%-11.5 \%$ compared to the normal population and the severity of depression and exacerbation of SLE rises the depression among SLE patients (3). Systemic sclerosis (SSc) is an autoimmune connective tissue disorder that is diagnosed by the fibrosis of skin, internal organs including the heart, lung, kidney, and digestive system and vascular demyelination. SSc can cause several painful symptoms, including endodontic ulcers, Raynaud's phenomenon, skin clefts, joint contraction and gastro-esophageal reflux (4).

\section{Objectives}

In this study: firstly, determining the prevalence of depression in common rheumatic diseases, so that, due to the importance of depression in these patients, psychiatric disorders can be detected early and suitable remedial measures can be done faster. The result can be useful in clinical management, and secondly, to find the association be- 
tween depression and personality traits, quality of life, social support, and the characteristics of these diseases and the rheumatologist will pay special attention to these complications and determine the risk of depression factors in rheumatologic patients.

\section{Methods}

\subsection{Data Collection}

The study population was the patients who referred to the rheumatology clinic of Golestan Hospital of Ahwaz in 2016.

Inclusion criteria: all patients were selected based on the American College of Rheumatology (ACR) criteria by a rheumatologist. The minimum age for the patients was 10 years.

Exclusion criteria: patents with coexisting disease that affects the main disease such as infections or heart disease, respiratory tract, gastrointestinal tract, nervous and ganglia diseases, patients with severe cognitive impairment, patients with significant hearing impairment, the lack of communication skills to ensure the test scores validation, patients who were illiterate.

\subsection{Depression Criteria}

To diagnose depression, there are two important ICD10 and DSM- 5 classification systems. To diagnose depression based on ICD-10, there must be four symptoms of depression and at least five symptoms of depression must be diagnosed in order to diagnose depression in the DSM-5. Of course, in both systems, these symptoms should be at least two weeks and clinically detrimental to the daily functioning of life, and one symptom in DSM-5 system or two symptoms in ICD-10 should be present in the symptoms of the patient. The main thing that involves depressed mood is the loss of interest or desire and energy. DSM-5 and ICD-10 use similar criteria, but they may differ in the diagnosis of those who fit the criteria (1).

\subsection{Screening Depression}

Commonly we used questionnaires such as PHQ-9, PHQ-2, BDI-PC and WHO-5 for screening depression (5, 6).

\subsection{Clinical Characteristics}

Depressed mood is a key symptom of depression. The patient may say that he feels sad, desperate, or unprotected. About two-thirds of all depressed patients are considering suicide and about $10 \%-15 \%$ of them commit suicide. Almost all depressed patients (about 97\%) are lagging behind their energy, which makes them unable to perform their duties easily, disrupts their academic and occupational skills and motivation to accept the new plans.
Other vegetarian symptoms of depression are menstrual irregularity and reduced sexual interest and function.

\subsection{Diagnostic Evaluations}

Patients with depression syndromes may refer to the physician with neurovegetative, cognitive, and physical symptoms. Dementia includes mood, emotional discomfort, emotional numbness, and sometimes anxiety or irritability. Neurovegetative symptoms include: loss of energy, change in sleep, appetite or weight. Some patients are more likely to experience physical symptoms (headache, abdominal or pelvic pain, back pain or other physical complaints) that can disturb depression. Pregnant women, elderly people, prisoners, some ethnic minorities, and lowincome patients are often overwhelmed by physical symptoms.

\subsection{Psychological Examination}

In assessing a patient suspected of depression one must particularly seek information regarding:

1. Disturbance in emotion.

2. Considering thoughts about depression in the patient.

3. Mental movement changes.

\subsection{Sample Size}

The sample size was evaluated with a 95\% confidence and (0.05) accuracy, and the prevalence of depression in the least-developed disease which was lupus erythematosus, the sample size was evaluated as 354 .

$$
N=\frac{Z^{2} p(1-p)}{d^{2}}=\frac{(1.96)(0.33)(0.67)}{(0.05)^{2}}=354
$$

\subsection{Analytic Tests}

Chi-square tests were used to compare the prevalence of depression in three diseases as well as the correlation ( $\alpha$ $=0 / 05$ ) and the statistical analysis were done by the 22nd version of the SPSS.

\subsection{Ethical Consideration}

This work was done under ethical considerations of Ahvaz Jundishapur University of Medical Sciences. 


\section{Results}

A total of 305 individuals entered the study out of the 354 participants according to inclusion and exclusion criteria. The mean age of the participants was 42.35 years, the minimum age was 12 and the maximum 80 years in the study, which was related to RA disease. 305 patients were female and $14.4 \%$ (44 patients) were male, indicating that most participants in this study were female. The mean of the disease period was 5.23 years, with a minimum of 1 year and a maximum of 42 years. Regarding education, $16.1 \%$ (49 patients) were illiterate, $25.6 \%$ (78 patients) were under diploma, 33.1\% (101 patients) had a high school diploma, 25.2\% (77 patients) had higher than diploma education, which indicates that most participants education were diploma education (see Figure 1).

76.1\% (232 patients out of 305 patients) were RA patients, $22.3 \%$ (68 patients) were SLE and 1.6\% (5 patients) were SSc. this study suggests that most patients had were RA patients (see Figure 2). 97.2\% (139 patients) out of 143 RA patients with depression used corticosteroid and 2.8\% (4 patients) had no use of corticosteroid. The results showed a significant relationship between depression and corticosteroid consumption $(\mathrm{P}<0.05)$, this means depression is directly related to corticosteroid consumption. 100\% (50 patients) of 50 SLE patients with depression used corticosteroids. The results showed that there is a significant relationship between depression and corticosteroid consumption $(\mathrm{P}<0.05)$ which means depression is directly related to corticosteroid use. $100 \%$ (3 patients) out of 3 SSc patients with depression consumed corticosteroids. The results showed a significant relationship between depression and corticosteroid consumption $(\mathrm{P}<0.05)$ which means depression is directly related to corticosteroid use. $96.39 \%$ (294 patients) consumed corticosteroids and 3.6\% (11 patients) did not use corticosteroids, which suggests that the

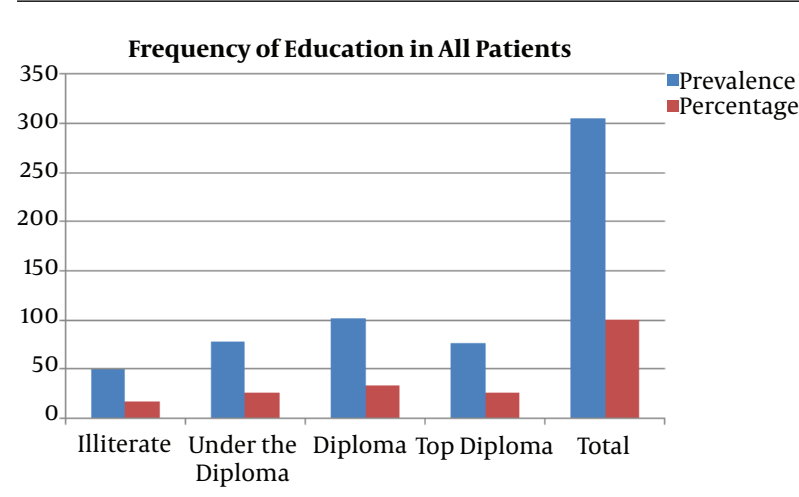

Figure 1. Frequency of education in all patients majority of patients used corticosteroids. 35.7\% (109 patients) had no depression and 18\% (55 patients) had mild depression, 28.5\% (87 patients) had moderate depression and $17.7 \%$ (54 patients) had severe depression, indicating that about one third of the patients were not depressed and the highest percentage of depression was moderate depression. Quality of life, physical health, mental health, social relationships, environmental health, and general health of these patients were $44.43 \%, 53.22 \%, 54.31 \%, 54.02 \%$ and $48.88 \%$, respectively. Figure 3 indicates that the majority of patients had moderate quality of life in all aspects.

The average social support score was 16.11, with the lowest score of 2 (the lowest social support) and the highest score was 23 (the most social support). According to the study, 38.36\% (89 patients) of RA patients had no depression and $17.67 \%$ (41 patients) had mild depression, $26.29 \%$ (61 patients) had moderate depression and 17.67\% (41 patients) had severe depression. Social support was reported total score of 17; patients with mild, moderate and severe depression were 16,15 , and 15 score, respectively. In all of

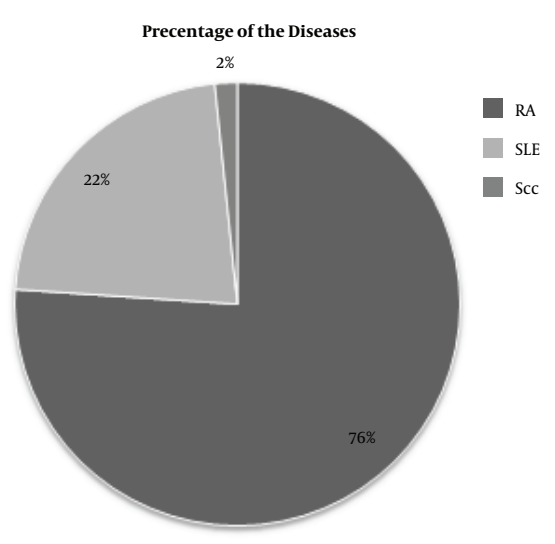

Figure 2. Frequency of disease type in the all patients

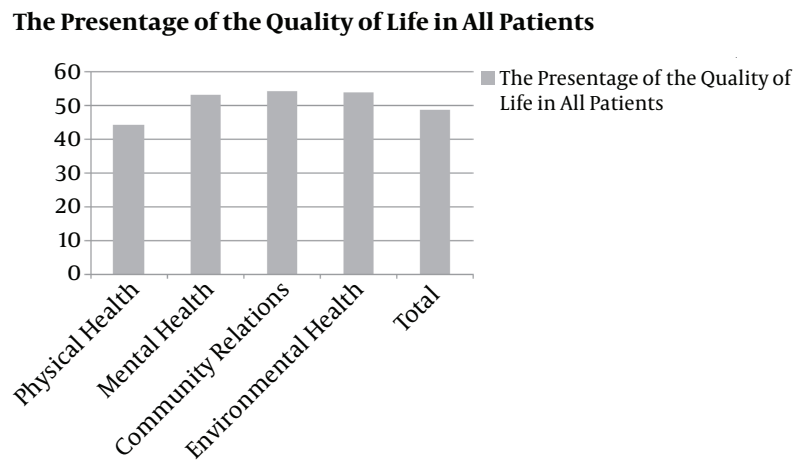

Figure 3. The percentage of the quality of life in all patients 
RA patients with depression, their social support score was 15.33. The results show a significant relationship between depression and social support $(\mathrm{P}<0.05)$, in other words, depression decrease the social support. Also there was a direct correlation between the severity of depression and the lack of social support (Figure 4).

According to the study on 68 patients with SLE, 26.48\% (18 patients) had no depression and 19.11\% (13 patients) had mild depression, 35.29\% (24 patients) had moderate depression and $19.11 \%$ (13 patients) had severe depression. The study shows the highest percentages of SLE were with moderate depression and mild to moderate depression had the same frequency (Figure 5).

According to the study, on SSc patients $40 \%$ (2 patients) had no depression and 20\% (1) had mild depression, 40\% (2 patients) had moderate depression, which suggests that patients with no depression and Also, patients with moderate depression have the same rates of depression and no patient had severe depression. In 232 patients with RA, the mean age of non-depressed, mild depression, moderate depression and severe depression was $44,45,45$, and 45 , respectively. The findings showed that there were no

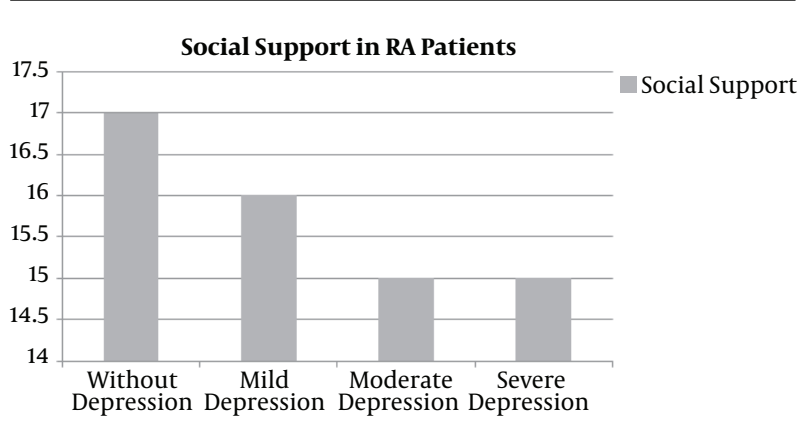

Figure 4. Social support in RA patients

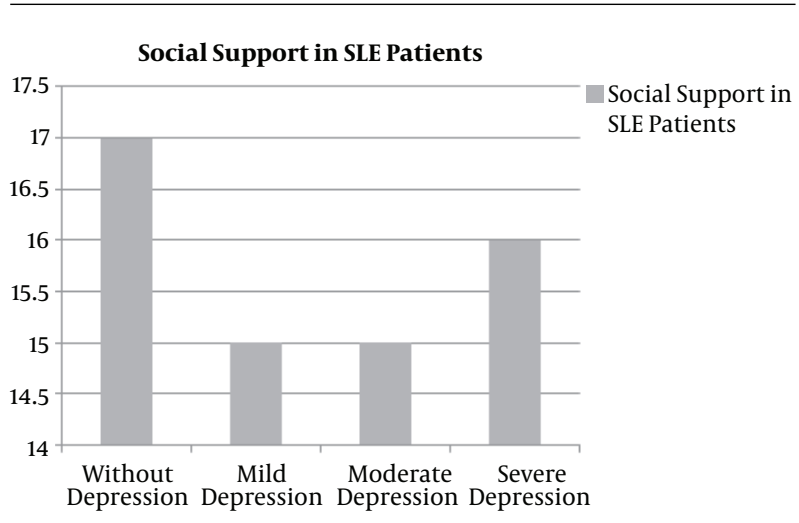

Figure 5. Social support in SLE patients significant difference between depression and age of RA in the patients. In 68 SLE patients, the mean age of nondepressed, mild depression, moderate depression and severe depression was 34,31,37, and 36, respectively. The findings showed that there was no significant difference between depression and age of patients. In 5 patients with SSc, the mean age of non-depressed, mild depressed and moderate depression was 48,27 , and 28 , respectively. The findings showed a significant difference between depression and the age of patients with SSc. depression had a reverse relationship with the age of patients. Totally, $83.91 \%$ (120 female patients) out of 143 RA patients were reported to be depressed and $16.08 \%$ (23 male) reported as depression cases. Results showed that there is a significant relationship between depression and gender in RA patients ( $\mathrm{P}$ $<0.05$ ). 94\% (47 female) and 6\% (3 male) out of the 50 patients with SLE were reported with depression. The evaluation shows that there is a significant relation between depression and gender in SLE patients $(\mathrm{P}<0.05)$. It was also observed that there is a correlation between the severity of the disease and depression in RA patients. In SLE patients with depression, the average percentage of quality of life in terms of physical health, mental health, community relations and environmental health were $42.66 \%, 51.66 \%, 52.33 \%$ and $52 \%$, respectively. The results showed a significant relationship between depression and quality of life $(\mathrm{P}<0.05)$, which means that depression has a relationship with the quality of life and associated with health. It was also observed that there is a reverse correlation between severity of depression in patients with SLE and quality of life related to health see. In the SSc patients with depression, the average percentage of quality of life in terms of physical health, mental health, community relations and environmental health were $37 \%, 49 \%, 45.5 \%$ and $59.5 \%$, respectively. The results showed a significant relationship between depression and quality of life $(\mathrm{P}<0.05)$.

\section{Discussion}

Depression can be associated with other medical problems, such as diabetes, heart disease, cancer, RA and many other diseases. The relationship between depression and diseases is complicated. Depression can be worsened when with medical problems, while it also can increase physical disease. Depression makes difficulties for the patient to manage his disease. Many biological, psychological, and social factors can interact with the development of depression (7). Serotonin monoamines, noradrenaline and dopamine are the main neurotransmitters in modulating the mood and behavior. Dysfunction in serotonergic and noradrenaline neurotransmitters and respectively dopamine decrease in depression. Anti-depressants that 
target serotonin and norepinephrine improve depression symptoms so there is a link between the interstitial nerves and depression. Dysfunction of other neurotransmitters such as gamma-aminobutyric acid (GABA) and glutamate, are also associated with depression. All of these neurotransmitters have a massive effect on the frontal cortex. Although functional impairment has been shown in the transmission of monoaminergic neurotransmitters in depression, it is not completely clear how this dysfunction develops in the nervous system $(8,9)$. In a study by Sruamsiri et al. 500 RA patients with demographic characteristics were studied, 176 patients (35\%) had depression symptoms but only 25 (5\%) were officially diagnosed or physician visits them due to depression (10). In the study done by Yilmaz et al., the prevalence of depression was reported $39.4 \%$ in RA patients (11), other studies of the prevalence of depression in RA patient by da Costa et al. (12), and Mella et al. (13) were reported 33.7\% and 53.2\% respectively. Raafat et al. reported a study on of 50 SLE patients, 64\% (32 patients) had showed one major symptom of depression according to depression questionnaire, while in the control group, 36\% (16 out of 50) showed depression symptoms (14). Schoenherr et al. reported that depressed mood correlates with the type of medicine taken by patients, for example corticosteroids increase the depression and this is consistent with our results. Therefore, physicians should consider depression among RA patients (15).

\subsection{Implication of the Study}

Regarding the high prevalence of depression in patients with rheumatoid diseases, rheumatologists should be given special attention to this issue, and psychiatric visits should be considered in the treatment of these patients, as well as medications that cause depression and this information is used in the treatment of rheumatic diseases.

\subsection{Conclusions}

We conclude that the prevalence of depression in RA, SLE and SSc patients were $61.63 \%, 73.52 \%$ and $60 \%$, respectively. There was no significant association between depression and age of patients in RA and SLE. There was a significant association between depression and gender in patients with SLE and RA, but the total number of SSc patients who were included in the study were low so we could not evaluate it in them. There was no significant association between depression and educational level in RA patients, while there is a significant association between depression and educational level in SLE patients. There was a significant association between depression and quality of life in all patients enrolled in this study. There was a significant association between depression and social support in all patients enrolled in the study. There was a significant association between depression and corticosteroid consumption in all patients enrolled in the study. There was a significant association between the disease duration and depression in patients with RA and SLE, while there was no significant association between the duration of the disease and depression in patients with SSc. Due to the prevalence of depression in patients with rheumatic diseases such as RA, SLE and SSc the rheumatologists should pay special attention to depression among his patients and consult with a psychiatrist in such cases, also in the case of the medicines such as corticosteroids that cause depression, the rheumatologist should consider the psychiatrist's advice. Finally, it is suggested to increase the information and the awareness of the patients and their family in psychological field and consider the role of family and friends in increasing the patients' social supports in order to reduce the level of the depression and the risk of suicide among the patients.

\section{Acknowledgments}

We wish to all our collagenous in Ahvaz Jundishapur University of Medical Sciences.

\section{Footnotes}

Authors' Contribution: all authors worked equally together.

Conflict of Interests: It is not declared by the authors.

Ethical Approval: This work was done under ethical considerations of Ahvaz Jundishapur University of Medical Sciences.

Funding/Support: Threr is no funding/support.

\section{References}

1. American Psychiatric Association. Diagnostic and statistical manual of mental disorders. 5th ed. Washington, DC; 2013. doi: 10.1176/appi.books.9780890425596.

2. Rajaei E, Haybar H, Mowla K, Zayeri ZD. Metformin one in a million efficient medicines for rheumatoid arthritis complications: Inflammation, osteoblastogenesis, cardiovascular disease, malignancies. Curr Rheumatol Rev. 2019;15(2):116-22. doi: 10.2174/1573397114666180717145745. [PubMed: 30019648].

3. Shen B, Tan W, Feng G, He Y, Liu J, Chen W, et al. The correlations of disease activity, socioeconomic status, quality of life, and depression/anxiety in Chinese patients with systemic lupus erythematosus. Clin Dev Immunol. 2013;2013:270878. doi: 10.1155/2013/270878. [PubMed: 23864877]. [PubMed Central: PMC3705842].

4. Benrud-Larson LM, Haythornthwaite JA, Heinberg LJ, Boling C, Reed $\mathrm{J}$, White $\mathrm{B}$, et al. The impact of pain and symptoms of depression in scleroderma. Pain. 2002;95(3):267-75. [PubMed: 11839426]. 
5. Pignone MP, Gaynes BN, Rushton JL, Burchell CM, Orleans CT, Mulrow $\mathrm{CD}$, et al. Screening for depression in adults: A summary of the evidence for the U.S. Preventive Services Task Force. Ann Intern Med. 2002;136(10):765-76. [PubMed: 12020146].

6. Arroll B, Khin N, Kerse N. Screening for depression in primary care with two verbally asked questions: cross sectional study. BMJ. 2003;327(7424):1144-6. doi: 10.1136/bmj.327.7424.1144. [PubMed: 14615341]. [PubMed Central: PMC261815].

7. Blazer DG. Depression in late life: Review and commentary. J GerontolA Biol Sci Med Sci.2003;58(3):249-65. doi:10.1093/gerona/58.3.m249. [PubMed: 12634292].

8. Dening T, Thomas A. Oxford textbook of old age psychiatry. 1. 2nd ed. Oxford University Press; 2013. doi: 10.1093/med/9780199644957.001.0001.

9. Nobile M, Begni B, Giorda R, Frigerio A, Marino C, Molteni M, et al. Effects of serotonin transporter promoter genotype on platelet serotonin transporter functionality in depressed children and adolescents. J Am Acad Child Adolesc Psychiatry. 1999;38(11):1396-402. doi: 10.1097/00004583-199911000-00014. [PubMed: 10560226].

10. Guelfucci F, Kaneko Y, Mahlich J, Sruamsiri R. Cost of depression in Japanese patients with rheumatoid arthritis: Evidence from admin- istrative data. Rheumatol Ther.2018;5(1):171-83. doi: 10.1007/s40744-0180096-4. [PubMed: 29470833]. [PubMed Central: PMC5935627].

11. Yilmaz H, Karaca G, Demir Polat HA, Akkurt HE. Comparison between depression levels of women with knee osteoarthritis, rheumatoid arthritis, and fibromyalgia syndrome: A controlled study. Turk J Phys Med Rehabil. 2015;61(3):197-202. doi:10.5152/tftrd.2015.87894.

12. da Costa AFC, Brasil MAA, Papi JA, de Azevedo MNL. [Depression, anxiety, and disease activity in rheumatoid arthritis]. Revista Brasileira de Reumatologia. 2008;48(1). Portuguese. doi: 10.1590/s048250042008000100003.

13. Mella LF, Bertolo MB, Dalgalarrondo P. Depressive symptoms in rheumatoid arthritis. Braz J Psychiatry. 2010;32(3):257-63. [PubMed: 20694442].

14. Raafat HA, El Refai RM, Alrasheed HA, El Din MN. Major depression and disease activity among systemic lupus erythematosus Egyptian females. Egypt Rheumatol. 2015;37(4):S1-6. doi:10.1016/j.ejr.2015.09.007.

15. Schoenherr SJ, Brown RT, Baldwin K, Kaslow NJ. Attributional styles and psychopathology in pediatric chronic -illness.J Clin Child Psychol. 1992;21(4):380-7. doi:10.1207/s15374424jccp2104_8. 